\title{
Standard Life
}

\author{
Lise Harou
}

\begin{abstract}
Standard Life
Against the dark and uniform background of "standard life" and its bureaucratic indifference, rises the luminous figure of Alexandra, fleeting subject of a discourse that seeks via desire and dream to pierce the screen of melancholy. Say " $I$ " and "you," imagine an encounter, suggests Harou, and transform the world...
\end{abstract}

Art is giving and sharing with others the most precious part of ourselves, which is our blood. - Yoko Ono

Cet hiver-là, dans les jours précédant la Saint-Sylvestre, le mercure descendit au-delà de -30 , d'où des records de température de -60 la nuit par grand vent. Les guirlandes lumineuses faisaient un peu oublier la dépression économique, pendant des autres dépressions.

Alexandra partit pour la Norvège où l'attendait sa vieille maman. Les routes et autoroutes faisaient penser à des pistes de motoneige. Cing morts en quelques heures. Même la Virginie était touchée. Kashtin, dans la salle à manger, faisait le portrait d'une enfance. La leur à l'un ou à l'autre? Ou peut-être une autre. Une petite fille, née aux alentours du 15 janvier, commençait à respirer, à boire et à courir. Un Brésilien qui voulait «changer de vie» était trouvé mort dans la soute d'un avion. Alexandre Felipe da Silva aura connu la triste célébrité d'un passager clandestin. Mais sans doute pas l'issue de ses rêves d'innovation.

Qu'adviendra-t-il de toi, Alexandra? De tes propres rêves? Toi aussi tu respires, tu prends tes repas à heures fixes, tu travailles et tu dors. Mais je ne voudrais pas que l'âge vienne briser ton ardeur. Tu es trop belle pour moisir, trop vive pour te réfugier toujours dans les sentiers battus. Autrement, tu perdras cette splendeur qui ne passe pas inaperçue, laisse un vide derrière elle quand tu te fais rare. Je dis «tu» et je dis «je», car pour avancer et pour agir, il faut être au coeur de l'interlocution intime, en 
établir au moins les virtualités, pour ensuite être capable de penser et de travailler tranquille.

Enfin, le rêve va percer l'épaisseur de la morosité.

Il y aura aussi cette mise en scène cruelle un soir dans la voiture : un scénariste de la radio a eul'idée d'utiliser de la guitare hawaïenne comme fond sonore. «J'ai horreur du temps des Fêtes», avait précisé Alexandra.

Le Fêtes ont passé. Puis le carnaval. Et il y eut des avions, des tonnes de neige, des températures polaires. Mais, au moins, Corinne D. Maillet était avec moi depuis qu'elle m'avait légué, sans le savoir, ses quatre visons griffus. Morte depuis longtemps peut-être Corinne D. Maillet. Un défunt phoque transformé en chapeau se ligue avec les quatre visons pour me protéger du froid. «Un héritage?» demandait quelqu'un. «Non. Une vente de garage.» On dit «froid sibérien» alors qu'il paraît que la Sibérie est l'une des voies d'avenir de l'humanité, un espace qu'il va falloir apprivoiser et habiter. Vas-tu me donner rendez-vous avant cela, Alexandra?

Il faut occuper les territoires pas encore marqués. Créer l'espace mental propice à la réappropriation de soi, à l'avancée vers l'autre. Écouter Léa Pool, Henri Solinas. Lire celles qui ont écrit, qui se sont donné la peine de penser et d'écrire. Probyn, Causse, Blais, Mailhot, Escomel, les grandes Françaises, Américaines, Britanniques et Américaines du Nord et du Sud qui les ont précédées. Explorer le plus sérieusement du monde le patrimoine féminin - matrimoine, disent certaines - de l'humanité. Faire vite parce qu'il y a trop de malades. Parce que l'inanition et l'aliénation continuent de guetter. De plus en plus incurables et engendrées ou renforcées par le système. Un système sûr de ses certitudes, qui voudrait s'autolégitimer et se suffire à lui-même, produire les statistiques de son autosatisfaction.

Dans les chemins sinueux et imprévisibles d'un désir dont la réciprocité reste hautement improbable, je te trouve tôt ou tard aux carrefours, Alexandra. Sous la figure de la skieuse de nuit des Plaines d'Abraham, un 5 février. À travers les intonations de H. Levitt qui raconte des histoires. Partout: dans les villes, sur les écrans de la pensée, dans les ornières du quotidien.

À la télévision, L'insoutenable légèreté de l'être s'éteint dans un brouillard meurtrier. Dans le brouillard de ce qui me reste de force de l'âge, je 
te désire ardemment, Alexandra, doucement, paradoxalement. La skieuse glisse sur la neige le long du fleuve, derrière la clôture de fer forgé de la rue de Bernières, sous des lampadaires anciens.

Au coin de l'avenue Briand, s'ouvre dans le noir la voûte des grands arbres, là où les jeunes femmes, le jour, promènent leurs enfants emmaillotés. Là où moi aussi je promenais les miens à leur âge. De plus en plus audacieuse dans mes rêves de toi, j'apprends petit à petit à te toucher. $\grave{A}$ distance d'abord, puis jusqu'à bouche que veux-tu. Je finis par te prendre avec moi sous la couette, sous le nuage du duvet. Et c'est alors seulement que je me souviens avec clarté des pointes de diamant qui viennent à la rencontre du pare-brise sur les routes de nuit. De la brillance des collines depuis lesquelles on s'élance vertigineusement, sur les pentes de ski, entre des sapins qu'on ne reverra plus jamais de toute façon.

***

Apprendre le tango, lechinois, l'arabe. En attendant de mettre ces projets à exécution, les cultiver en rêve, trouver des partitions, avoir l'oeil ouvert, l'oreille tendue, inspirée par cette pianiste sud-américaine qui, en dépit de vertèbres brisées dans un accident et défiant les pronostics de ses médecins, avait continué à jouer.

Tu m'avais reproché un jour, Alexandra, d'être «trop conceptuelle». Était-ce une manière d'évoquer ces rêves réalisables auxquels j'aime m'attacher comme à une bouée pour les mauvais jours? Tu me soutiens à distance à la manière de ces potions vitaminées dont l'effet, pour invisible qu'il soit à l'oeil nu, n'est pas moins radical. L'hiver est sale, interminable. Je rêve de ta fine écriture bleue, de ta voiture bleue, de la mer presque verte où tu viendrais peut-être te reposer avec moi, un jour que le fardeau du temps se serait de toute façon déposé sur tout ce qui nous est cher.

Les pigeons sont attablés devant la station de métro Côte-SainteCatherine, le long de la clôture de fer forgé. Il fait soleil. Un ouvrier prend son repas de midi assis sur une planche dans le chantier de dynamitage de la Côte-des-Neiges. La rue Victoria m'appelle, avec ses couleurs chaudes, ses boissons exotiques, et surtout, surtout, ses visages de toutes les origines, paisiblement réunis. Mais l'autobus m'emporte ailleurs, là où le devoir se tient, là où les gens qui font quelque chose se tiennent. $\mathrm{Ou}$ plutôt sont tenus par l'ordre établi qui définit aussi bien leurs habitudes vestimentaires que leur horaire, emploi du temps, lieu d'assignation et rayon d'action. Qui disait que le monde meurt à force d'indifférence? 
Qui plus est, il a été pensé pour l'indifférence, l'interchangeabilité. Tout le contraire de l'interaction, avec les risques qu'elle comporte. Le facteur humain, infailliblement porteur d'un minimum d'imagination et de liberté, jamais complètement prévisible, c'est le germe de la terreur au sein de la technocratie. Et c'est la peur d'un potentiel élément perturbateur qui fait qu'on. lutte avec acharnement contre le changement, quel qu'il soit, en faisant semblant de le rechercher, pourchassant avec l'énergie du désespoir le grain de sable indésiré qui risque de s'introduire dans l'engrenage aveugle, exigeant du connu, rien que du connu. «In fact, I believe that professionalism kills art», dit Yoko Ono à Eva Zanardi. Vice Versa transporte cette parole pleine de sagesse jusqu'à nous. C'est elle aussi qui parle de «daily struggle of ordinary people trying to survive in a violent societys.

«La vie n'est courte», écrivait Sénèque, «que par le mauvais emploi $q u^{\prime}$ on en fait». Et par ailleurs Virgile:

Les meilleurs jours de la vie des malheureux mortels

Sont toujours les premiers à s'enfuir.

Pourquoi attends-tu? dit-il, pourquoi restes-tu sans rien faire? Si tu ne $t^{\prime}$ en saisis pas, ils s'enfuient.

Et même si tu t'en saisis, répond Sénèque, ils s'enfuiront; aussi fautil opposer à la rapidité du temps la vitesse avec laquelle on en use et y boire promptement commeà un tourment impétueux aux eaux éphémères.

Tu as dû oublier ces vers, Alexandra. Et comme tu les as oubliés je suis entrée dans le labyrinthe par inadvertance. Dans les souterrains de Montréal : métro, universités, grands magasins, gares, c'est chaque jour un peu de l'univers d'Alice au pays des merveilles et un peu celui de «la maison des horreurs». Comme dans «la maison des horreurs» des foires, en effet, chaque angle, chaque surface peut faire surgir quelque chose d'inattendu censé vous prendre à la gorge. Au quotidien, les hôpitaux et la morgue font office de fils d'araignées et de masques de la turpitude. Les gens meurent comme des mouches, ou bien ils font de la tachycardie paroxystique, subissent des proliférations, des chutes ou des dérèglements spectaculaires, ou bien ils éprouvent des symptômes terribles. Il y a des cortèges funèbres et des visites à l'hôpital, où espoirs et désespoirs s'affrontent, se renforcent, se complètent. 
Et toi, Alexandra, tapie en tes refuges soigneusement ménagés au fil des ans, tu fais semblant de ne rien voir, de ne rien entendre, de ne rien apercevoir. Tu négocies tes échanges avec le monde de manière que soit préservée à tout prix ta tranquillité souveraine. Comme la paix de tous les royaumes, la tienne est pourtant constamment menacée par des courants souterrains, par le tumulte intérieur contre lequel tu luttes avec courage. Comme si de rien n'était, bien entendu, et surtout à condition que nul ne l'évoque. As-tu seulement vu qu'il y a du soleil aujourd'hui? En regardant la neige sale, je pense à ton jardin peuplé de conifères, de branches et broussailles nues, mais sans doute habitées par quelques oiseaux quand il fait beau. Et ta présence invisible en cette vie me réchauffe imparfaitement le coeur, attise à la manière du petit bois encore vert le brasier de mes ardeurs.

Au comptoir alimentaire du quartier, deux Arabes, se croyant seuls, fredonnent une mélopée comme celles qu'Oum Khalsoum a rendues populaires. Toi tu regardes le jardin depuis ta chambre ou ton bureau, ou alors tu boudes dans la cuisine ou sous la douche ce ciel bleu qui n'a rien de bon à t'apporter pour aujourd'hui, crois-tu. Tu n'as vraisemblablement pas remarqué que ton existence avait le pouvoir de transformer la face du monde. 